\title{
Electrophysiological Studies with LON-954, a Tremorogen, in the Cat Spinal Cord
}

\author{
D.K. GANGULY* H.-G. ROSS and S. CLEVELAND \\ Physiologisches Institut (LST II). Universität Dusseldorf. Moorenstrasse 5. \\ 4000 Dusseldorf, Federal Republic of Germany \\ Accepted June 15, 1984
}

LON-954 [N-carbamoyl-2-(2,6-dichlorophenyl) acetamidine hydrochloride] has been reported to possess a tremorogenic property comparable to that of oxotremorine, the well known tremorogenic agent (1). The pharmacological profile of LON-954 indicated that it could be a more appropriate tool than oxotremorine for evaluation of antitremor drugs which may act through dopaminergic mechanisms (1)

We earlier reported the involvement of the skeletomotor apparatus and the spinal cord in oxotremorine-induced tremor (for references, see 2, 3). Recently, we have demonstrated that LON-954 also affected the skeletomotor site (4), and here, we report the effects of LON-954 on the motor output and discharges of Renshaw cells in the cat spinal cord.

The details of the methods were described in an earlier paper (2). In brief, the experiments were carried out either in anaesthetized (pentobarbitone sodium, $40 \mathrm{mg} / \mathrm{kg}$, i.p.) or intercollicularly decerebrated cats of either sex. Blood pressure was monitored throughout the experiment, and the body temperature was maintained between 37 and $39^{\circ} \mathrm{C}$. In experiments with decerebrated cats, all measurements were performed at least $2 \mathrm{hr}$ after decerebration and removal of halothane anaesthesia. The right hind limb was denervated as completely as possible, and the left dorsal roots L6 to $S 1$ were cut

* Dr. Ganguly received support from the Alexander Von Humboldt Fouridation. Bonn (Fed. Rep. Germány). Present address: Division of Pharmacology and Experimental Therapeutics, Indian Institute of Chemical Biology, 4, Raja S.C. Mullick Road, Calcutta 700032, India intradurally.

The discharges of the Renshaw cells from the segment L7 were recorded, after laminectomy, extracellularly through glass micropipettes filled with $3 \mathrm{M} \mathrm{KCl}$. The cells were identified by their monosynaptic burst response to single antidromic volleys. Antidromic stimulation of either the central end of the cut left ventral root $L 7$ or the left gastrocnemius were used for exciting the Renshaw cells. A single stimulus of $0.2 \mathrm{msec}$ duration at supramaximal strength for alpha fibers was applied every second, in some cases once in $3 \mathrm{sec}$.

Monosynaptic reflexes were elicited by single shocks applied to the central end of the cut dorsal root L7 or S1 and recorded from the gastrocnemius or anterior tibial nerves; their height was taken as a convenient measure of synchronus motor activity. Stimuli were applied every 10 or $15 \mathrm{sec}$ and were submaximal as judged by the reflex amplitude during the control period.

LON-954, when injected intraperitoneally ( $5 \mathrm{mg} / \mathrm{kg}$ ) in two intact (unanaesthetised) cats in order to observe its gross behavioural effects, caused a rest tremor which began 5 to $7 \mathrm{~min}$ after injection and was maximal within 15 to $30 \mathrm{~min}$. Signs of sympathetic overactivation: marked piloerection, dilatation of the pupils and aggressive behaviour were prominent in these animals.

It was observed that an intravenous dose of $2.5 \mathrm{mg} / \mathrm{kg}$ of LON-954 produced an effect that was comparable to the earlier reported effect of oxotremorine on the discharges of antidromically activated Renshaw cells (2). Hence, this dose of LON954 was selected to examine the spinal effects of the drug throughout the course of 
the study. In three intercollicular decerebrate cats, LON-954 (2.5 mg/kg, i.v.) produced irregular and jerky movements of the limbs, but there was no noticeable tremor. Electromyographic activity in these cats was considerably increased in the biceps and triceps brachii muscles following drug injection. No alternating bursts were observed in the electromyograms from these antagonistic muscles.

Intravenous injection of LON-954 (2.5 $\mathrm{mg} / \mathrm{kg}$ ) consistently depressed the height of the monosynaptic reflex recorded in the gastrocnemius nerve after electrical stimulation of the central end of the cut dorsal root ( $L 7$ or S1) in both pentobarbitone anaesthetized (2 experiments) and decerebrate (2 experiments) cats. A representative experiment is illustrated in Fig. 1a. The depressant effect of the drug was almost immediate, and recovery occurred after 60 to $90 \mathrm{~min}$.

Following administration of LON-954 (2.5 $\mathrm{mg} / \mathrm{kg}$, i.v.), Renshaw cells responded to single antidromic stimuli to ventral roots with an increased number of spike discharges. Again, this is true for anaesthetized ( 3 experiments) and decerebrated (2 experiments) animals. As may be seen from Fig. 1b, both the impulse frequency within a burst discharge and its duration were increased by the drug. The onset of LON-954 action was within 7 to $10 \mathrm{~min}$ and lasted for 60 to $90 \mathrm{~min}$, the maximal effect being at 15 to $30 \mathrm{~min}$ after injection (comparable to the overt motor disturbance caused by the drug). LON-954 also had a marked facilitatory effect on the late responses of Renshaw cells (Fig. 2), and their spontaneous activity was increased significantly under its influence. Under control condition the late responses did not appear after $1 \mathrm{sec}$ of antidromic stimulation.

The increase in spontaneous activity of the Renshaw cells under the influence of LON954 is unlikely to be secondary to enhanced motoneuron excitability since the monosynaptic reflex was consistently depressed by the drug. Since LON-954 did not produce any appreciable change of blood pressure after intravenous injection, any indirect effect on the spinal cord via vascular action can be excluded.
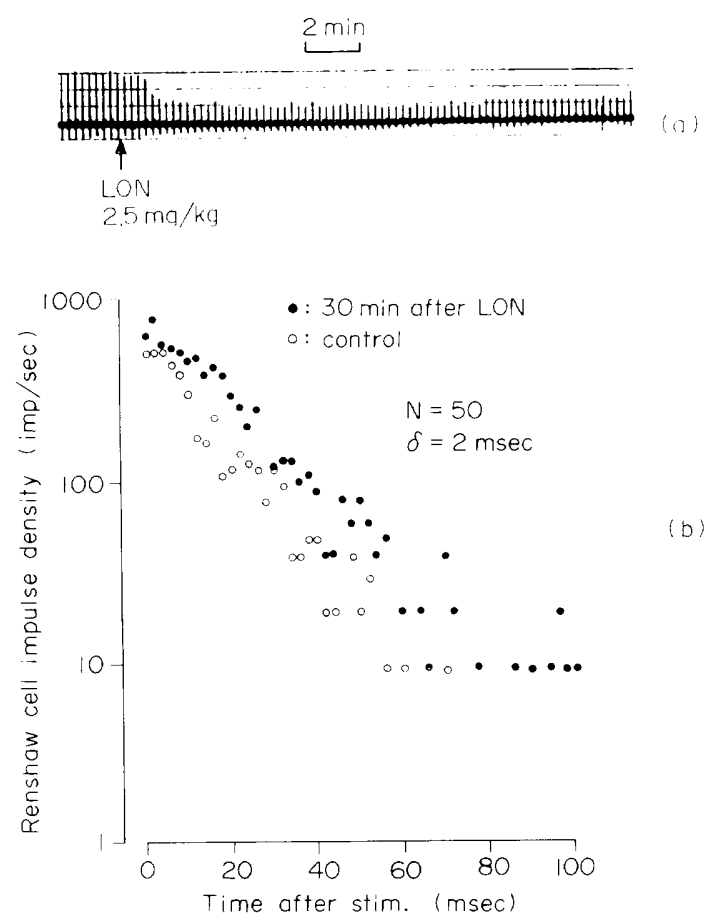

Fig. 1. a) Inhibition of monosynaptic reflex by LON-954 in a pentobarbitone anaesthetised cat. Reflex in tibials anterior nerve was elicited by shocks to dorsal root L7. At the arrow. $2.5 \mathrm{mg} / \mathrm{kg} \mathrm{LON-954}$ was administered intravenously. b) Decerebrate cat-average Renshaw cell activity before and after LON-954 (2.5 mg/kg, i.v.) administration: effect on burst response. Impulse density was measured in 2 msec counting intervals starting at zero time $(t=0)$ of a stimulus to ventral root $L 7$ (supramaximal stimuli, rate $1 / \mathrm{sec}$ ) and averaged over 50 trials each for the control (open circle) and beginning $30 \mathrm{~min}$ after LON-954 (closed circle). Note logarithmic ordinate.

Thus, the present experiments demonstrate that LON-954, like oxotremorine, affects several elements of the spinal motor system. While the drug did induce a sustained rest tremor in cats that were otherwise untreated, it failed to do so in decerebrate preparations, suggesting that higher structures of the CNS are necessary for tremorogenesis by LON-954, in contrast to the case of oxotremorine, which could generate tremor in intercollicularly decerebrate cats (3). This is in accord with the findings in rats (1) and supports the conclusion that tremor due to LON-954 may originate in an area rostral to 


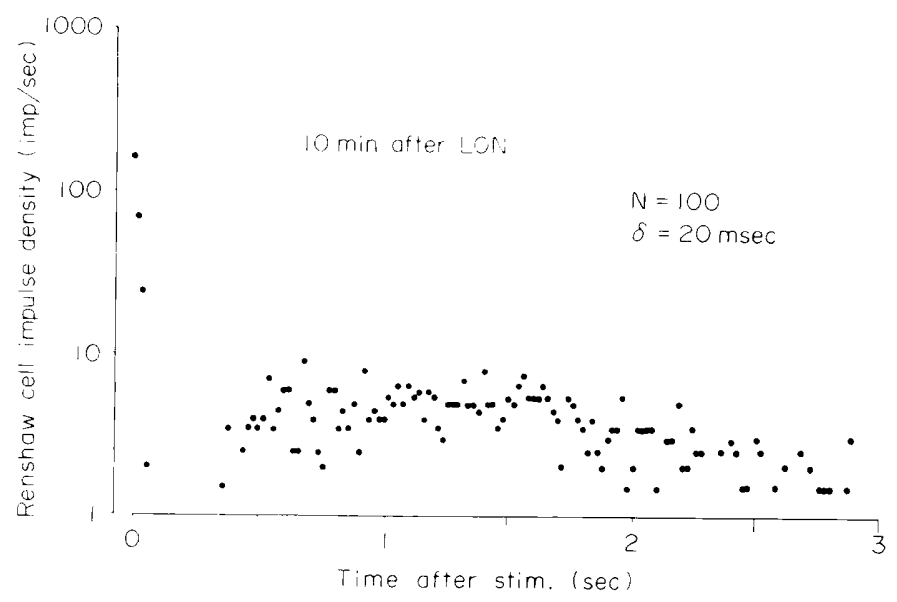

Fig. 2. Decerebrate cat-average Renshaw cell activity after LON-954 (2.5 mg/kg, i.v.) administration: effect on late response. Impulse density measured in 20 msec counting intervals starting at zero time $(\mathrm{t}=0)$ of a stimulus to ventral root $\mathrm{L} 7$ (supramaximal stimuli, rate $1 / 3 \mathrm{sec}$ ) and averaged over 100 trials beginning 10 min after LON-954

the inferior colliculi. It is also possible that an unknown amount of supraspinal influence might contribute in the spinal effects reported here, as in our experiments, the cats were decerebrated but not spinalised. The mechanism of action of LON-954 on the monosynaptic reflex and Renshaw cell excitability need not be simple, but such changes must affect motor performance, independent of the effects of the drug on higher centers of the central nervous system.

Antidopaminergic mechanisms have been implicated in the action of LON-954 on the basis that L-DOPA antagonizes its action. while pimozide, a dopamine receptor blocking agent, intensifies it (1). Such a mechanism may be involved in the facilitatory action of LON-954 on the cholinergic transmission to Renshaw cells since L-DOPA has been reported to inhibit the spike discharges of these interneurons elicited by antidromic stimulation (5). We also found in two anaesthetised cats that pimozide $(2 \mathrm{mg} / \mathrm{kg}$. i.v.) markedly increased the antidromically activated burst responses as well as the late responses of Renshaw cells. The spontaneous activity of the cells tested also increased under the influence of pimozide.

This effect of pimozide coupled with the reported effect of L-DOPA (5) on the discharges of the Renshaw cells strongly suggest the possibility of dopaminergic modulation of cholinergic neurotransmission from motor axon collaterals to Renshaw cells like in motor nerve terminals (6). In view of the postulated dopaminergic mechanism in the tremorogenic action of LON-954 (1), further studies on pharmacological interaction between LON-954 and dopaminergic agonists/antagonists on motor output and recurrent inhibition in spinal cats are warranted to implicate the spinal involvement in tremorogenesis by LON-954.

Acknowledgement: The authors are grateful to Dr A.C. Sayers of Research Institute Wander, Berne. Switzerland for the gift of LON-954.

\section{References}

1 Coward, D.M., Dogget, N.S. and Sayers, A.C.: The pharmacology of $N$-carbamoyl-2-(2.6dichlorophenyl) acetamidine $\mathrm{HCl}$ (LON-954). a new tremorogenic agent. Arzneimittelforsch. 27, 2326-2332 (1977)

2 Ganguly, D.K., Ross, H.-G., Cleveland, S. and Haase, J.: Effect of oxotremorine on the response of antidromically activated Renshaw cells in decerebrate cats. Exp. Brain Res. 25, 35-43 (1976)

3 Cleveland, S., Ross, H.-G., Ganguly, D.K. and Haase, J.: Generation of tremor in intercollicular decerebrate cats. Pflugers Arch. Supp. 373. R71 (1978)

4 Vedasiromoni, J.R. and Ganguly, D.K.: N- 
carbamoyl-2-(2.6-dichlorophenyl) acetamidine hydrochloride (LON-954), a tremorogen, on rat diaphragm. Japan. J. Pharmacol. 34, 353-355 (1984)

5 Meyer-Lohmann, J. Hellweg, G., Hagenat, R. and Bennecke, R.: Renshaw cells activity and morioamine liberation. Proc. Int. Union Physiol. Soc. 9, 389 (1971)

6 Ganguly, D.K. and Das, M.: Effects of oxotremorine demonstrate presynaptic muscarinic and dopaminergic receptors on motor nerve terminals. Nature 278, 645-646 (1979) 